\title{
ANALYSE DE L'IMPACT DE LA DIGUE D'AGRANDISSEMENT DU PORT DU MUSEL (GIJÓN, ESPAGNE) SUR LA DYNAMIQUE LITTORALE.
}

\author{
Nicolas Garcia, Raúl Medina, Pedro Lomónaco, Mauricio González \\ Groupe d'Ingénierie Océanographique et Côtière \\ Université de Cantabrie \\ Avenida de los Castros s/n, 39005 Santander, ESPAGNE \\ Tel. +34-942-201810, Fax +35-942-201860 \\ email: garciani@unican.es
}

\section{Résumé}

La construction de la digue Torres, digue d'agrandissement du port de Gijón, requiert une étude préalable des répercussions de l'ouvrage sur la morphodynamique des plages alentour. Cet article traite de la morphodynamique de la plage de San Lorenzo, directement affectée par la présence de la nouvelle digue : les changements induits dans la propagation de la houle, principalement une réduction de l'énergie incidente et la diffraction des fronts, se manifestent sur la plage par une augmentation de la pente du profil et une rotation de la ligne de côte dans le sens horaire. Un rechargement de sable permet d'exploiter ce changement de configuration pour augmenter la largeur de plage sèche, actuellement déficitaire dans la zone ouest, et d'améliorer ainsi la fonctionnalité de la plage.

\section{Abstract}

The construction of the Torres breakwater, as part of the expansion plan of the industrial port of Gijón, requires a previous evaluation of the morphodynamic impact of the structure on the beaches in the vicinity of the port. This paper contains the specific study of the San Lorenzo beach morphodynamic behaviour, directly affected by the presence of the new breakwater. The changes on the wave propagation, namely a reduction of the incident energy and the diffraction of the wave fronts, induce a beach profile steepening and a clockwise rotation of the coast line. This change of the beach configuration, coupled with a sand nourishment, would permit to overcome the current lack of dry beach, improving its functionality.

\section{Introduction}

Le port du Musel, port industriel de la ville de Gijón et l'un des plus importants d'Espagne, connaît aujourd'hui un problème de déficit de surface 
utilisable. L'Autorité Portuaire a décidé d'y remédier par la construction d'une nouvelle digue, appelée digue Torres, de $2 \mathrm{~km}$ de long, offrant 180 ha de surface de terrain. Compte tenu de la proximité des plages de Gijón, base du développement touristique de la ville, et des dimensions de l'ouvrage prévu, une étude préalable de son impact sur la dynamique littorale de la zone est indispensable. Le Musel compte cinq plages dans ses environs immédiats, les plages de Xivares, Arbeyal, Poniente, San Lorenzo et Peñarrubio (figure 1). Cet article traite de l'impact de la digue Torres sur la plus importante de ces cinq plages, en termes de fréquentation et de dimension, la plage de San Lorenzo.

L'étude réalisée utilise la méthodologie et les outils numériques développés pour ce type de projets par le Groupe d'Ingénierie Océanographique et Côtière (G.I.O.C.) de l'Université de Cantabrie. Elle consiste en une analyse des conditions d'équilibre de la plage en profil et en plan (analyse à long terme) et de son comportement face à des évènements de forte houle (analyse à court terme). L'équilibre à long terme est étudié à partir des formules de González et al. (2001) et Medina et al. (2000). L'analyse à court terme utilise une série de programmes, regroupés sous le nom de MOPLA, qui déterminent la houle irrégulière qui atteint la plage, le système de courants associés au déferlement des vagues puis le transport solide généré par ces courants, permettant ainsi d'évaluer les zones d'érosion et d'accumulation sur la plage et la variation de la bathymétrie. La structure de cet article suit les différentes étapes de la méthodologie mentionnée plus haut : description de la morphologie du site, étude de la dynamique marine (climat maritime, houle et systèmes de courants) et analyse de la morphodynamique de la plage, en situation actuelle puis postérieurement à la construction de l'ouvrage.

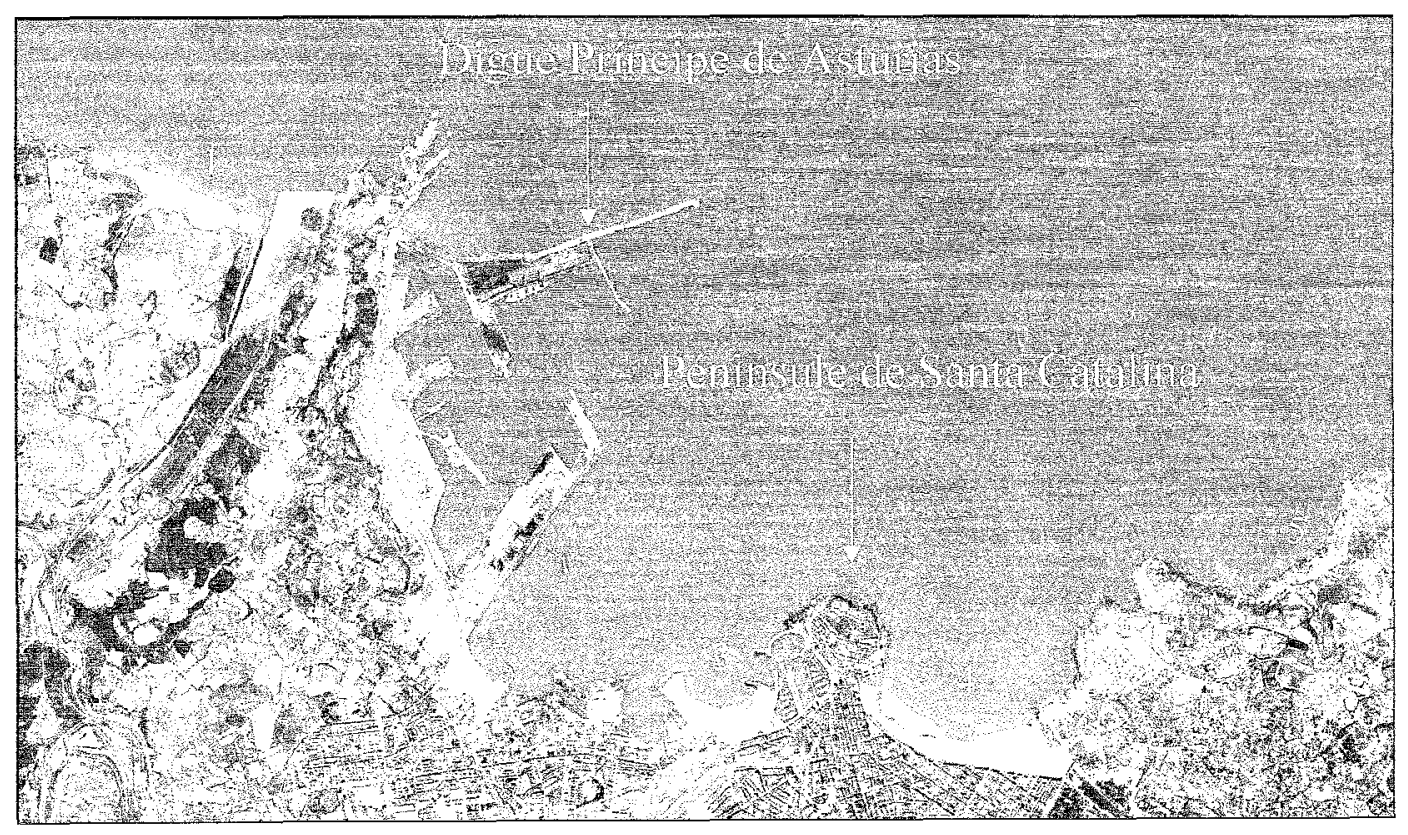

Figure 1. Port du Musel et plages de Gijón

Figure 1. Musel port and Gijón beaches

(1 Xivares 2 Arbeyal, 3 Poniente, 4 San Lorenzo, 5 Peñarrubia) 


\section{Morphologie de la zone d'étude}

Le port de Gijón est inclus dans l'unité physiographique délimitée par les deux grands caps que sont Cabo Peñas à l'ouest et Cabo San Lorenzo à l'est. Longue de $20 \mathrm{~km}$, l'unité se caractérise par un littoral de falaises très découpé, abritant de nombreuses petites plages. L'orientation N.-O./S.-E. de la côte et des lignes bathymétriques offre un premier abri au port du Musel face aux hoúles du N.-O., dominantes en Mer Cantabrique. D'autre part, les études géophysiques réalisées sur le site ont mis en évidence l'absence de dépôts de sable, dont la principale conséquence est une bathymétrie très irrégulière caractérisée par de nombreux hauts-fonds rocheux, comme le haut-fond des Amosucas. Au sein de cette unité, on peut distinguer des sous-unités physiographiques, indépendantes du point de vue sédimentaire, l'une d'elles étant la plage de San Lorenzo.

Dans sa configuration actuelle, la plage de San Lorenzo a une longueur totale de $1,3 \mathrm{~km}$. Elle est limitée au sud par le mur de soutènement de la promenade, à l'est par l'embouchure de la rivière Pilés et à l'ouest par le cordon littoral menant à la butte de Santa Catalina. La superficie de plage sèche, de $230000 \mathrm{~m}^{2}$ à marée basse, est réduite à $48000 \mathrm{~m}^{2}$ à marée haute, ce qui, compte tenu de la fréquentation de la plage en été, se révèle très insuffisant. Les fonds marins de l'anse de San Lorenzo se caractérisent par la présence d'un canal central perpendiculaire à la ligne de côte, constitué de sédiments sablonneux récents, et d'un substrat rocheux qui affleure à marée basse au niveau des extrémités est et ouest de la plage. La présence de ces zones rocheuses, comme nous le verrons plus loin, conditionne dans une large mesure la houle qui atteint la plage.

\section{Dynamique marine}

Cette partie a pour objectif la caractérisation de la houle et des systèmes de courants sur la plage de San Lorenzo à partir des données de climat maritime.

\subsection{Climat maritime}

Les données disponibles de houle pour la zone de Gijón proviennent de trois sources distinctes : données visuelles fournies par des observateurs entraînés à partir de bateaux parcourant les voies commerciales, données fournies par des modèles de génération de houle à partir de données météorologiques (projet WASA) et données instrumentales fournies par les balises du Programme de Climat Maritime de la Direction Générale des Ports.

L'information utilisée pour cette étude est résumée dans l'Atlas de Climat Maritime du Littoral Espagnol, publié par la Direction Générale des Ports. Cet atlas établit un découpage du littoral espagnol en 10 zones distinctes, définies à partir de leurs caractéristiques climatiques, de la configuration de la côte et de l'emplacement des instruments de mesure disponibles. Le port de Gijón est inclus dans la Zone I de l'Atlas, dans laquelle le climat maritime se caractérise essentiellement par la prédominance des houles du quadrant $\mathrm{N}$.-O., une hauteur de vague moyenne annuelle de $1 \mathrm{~m}$ et une $\mathrm{H}_{\mathrm{s} 12}$ de $5,7 \mathrm{~m}$. 


\section{VII ${ }^{\grave{m} e s}$ Journées Nationales Génie Civil - Génie Côtier, Anglet, France, 15-17 Mai 2002}

\subsection{Houle et courants sur la plage de San Lorenzo}

\subsubsection{Modèles utilisés}

La propagation des données de houle jusqu'à la plage objet de l'étude et la détermination du système de courants induits fournissent l'information nécessaire à l'analyse de la dynamique à court et à long terme. Le modèle de propagation utilisé dans cette étude est le modèle de propagation de houle irrégulière OLUCASP développé par le G.I.O.C. qui résout la forme parabolique de l'équation de la « mild-slope » et simule les phénomènes de réfraction, diffraction, réflexion, shoaling, déferlement des vagues et dissipation de l'énergie par frottement sur le fond. Au moyen de ce modèle a été réalisée la propagation depuis les eaux profondes jusqu'à la côte de huit spectres de houle «types», ayant des caractéristiques conformes aux indications de l'Atlas de Climat Maritime du Littoral Espagnol. Les systèmes de courants associés au déferlement ont ensuite été déterminés à l'aide du modèle COPLA-SP, modèle bidimensionnel résolvant les « shallow water equations », en incluant les termes de tenseur de radiation dû à la houle. Ces simulations ont permis de caractériser les patrons moyens et extrémaux de la dynamique de la plage (pour l'analyse à court terme)

D'autre part, à l'aide du modèle OLUCA-SP, ont été réalisées des épures de propagation de la houle depuis les profondeurs infinies jusqu'à la plage de San Lorenzo qui ont permis, à partir de la propagation de chacune des données de la base de données du projet WASA, de déterminer la direction du flux moyen d'énergie et les régimes moyens et extrémaux, informations nécessaires pour l'analyse à moyen et long terme.

\subsubsection{Résultats}

D'un point de vue général, la plage de San Lorenzo est protégée des houles du quatrième quadrant par le cap Peñas et la configuration général de la bathymétrie extérieure, ainsi que par la digue "Príncipe de Asturias", principale structure d'abri du Musel dans sa configuration actuelle. Il existe sur la plage un fort gradient latéral de hauteur de vague dû à linfluence du bas-fond des Amosucas. Il faut noter par ailleurs la forte influence des reliefs qui constituent les limites latérales de la plage et qui provoquent une concentration locale de l'énergie de la houle. Cette concentration locale est, avec le gradient longitudinal de hauteur de vague décrit précédemment, l'élément qui gouverne le système de courants associés au déferlement de la houle sur la plage de San Lorenzo.

$\mathrm{Ce}$ système de courants est constitué principalement de deux flux longitudinaux dirigés vers le centre de la plage, venant l'un de la péninsule de Santa Catalina, l'autre des falaises à l'est de la plage. Ces courants sont dus à la différence de hauteur de vague existant entre la zone centrale et les limites latérales de la plage où se concentre l'énergie incidente. La rencontre de ces deux courants de sens opposés génère, dans la zone centrale de la plage, un flux dirigé vers le large. La localisation du point de départ de ce flux varie en fonction de l'intensité des deux courants latéraux. Ce flux sortant est à l'origine, en période de forte houle, de l'accumulation de sable en forme de barre transversale visible à 
marée basse dans la zone ouest de la plage. Ce schéma de système circulatoire se produit, avec quelques légères variantes, quelles que soient la direction et la période de la houle incidente. Une augmentation de la hauteur de vague entraîne bien sûr une augmentation de l'intensité des courants.

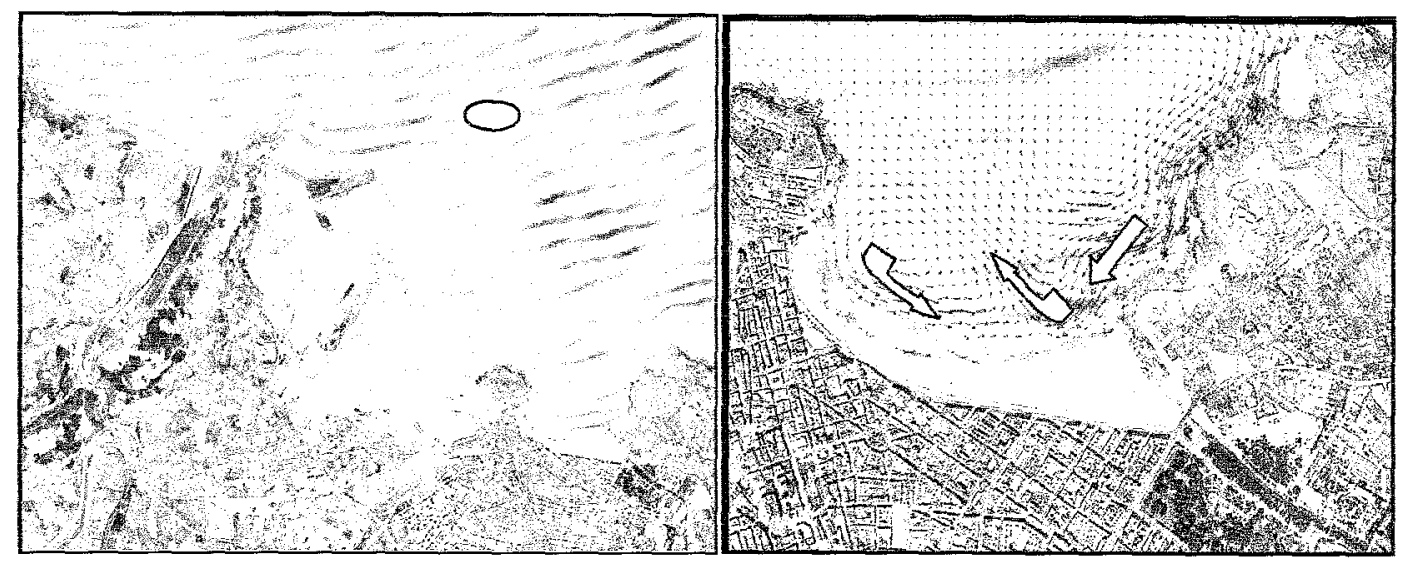

Figure 2. Exemple de propagation d'une houle du N.-O. et système de courants associé sur la plage de San Lorenzo

Figure 2. Example of $N W$ wave propagation and wave-induced currents on San Lorenzo beach

\section{Morphodynamique littorale actuelle}

Le but de cette partie est d'étudier la morphologie et la stabilité actuelle de la plage, afin d'évaluer ultérieurement les répercussions de la construction de la digue Torres sur cet équilibre. La stabilité du profil est analysée à partir du modèle biparabolique de profil d'équilibre de Medina et al. (2000) et l'étude de la stabilité en plan utilise la méthodologie de forme d'équilibre proposée par González (2001).

\subsection{Equilibre en profil}

Les relevés effectués lors de la campagne bathymétrique réalisée à San Lorenzo en octobre 1994 montrent l'existence pour un même profil de deux zones distinctes, l'une comprise entre les cotes de marée haute et de marée basse (profil d'estran), le marnage étant de $3,5 \mathrm{~m}$, et l'autre sous la cote de marée basse (profil immergé). Il apparaît par ailleurs un brusque changement de pente aux cotes -4 à $-5 \mathrm{~m}$, où le profil de sable intercepte le substrat rocheux. La comparaison des profils des différentes zones de la plage met en évidence que le profil d'estran, entre le mur et la cote 0 , a une longueur de $200 \mathrm{~m}$ approximativement. Cette partie du profil a des pentes distinctes en fonction de sa position, les profils les plus pentus étant ceux de la zone est où se trouvent précisément les sédiments les plus gros et où les courants sont les plus forts. En général, l'ajustement entre les profils théoriques obtenus à partir des formules de Medina et al. (2000) et les profils mesurés est bon (voir figure 3 ). Les valeurs du $D_{50}$ obtenues à partir des paramètres d'ajustement des parties émergées et immergées sont très proches des 
valeurs que donnent les échantillons prélevés sur la plage : $0,30 \mathrm{~mm}$ pour le profil d'estran et $0,25 \mathrm{~mm}$ pour le profil immergé dans la zone centrale de la plage.

\subsection{Equilibre en plan}

La méthodologie développée par González (2000) permet, à partir de la direction du flux moyen d'énergie $a u(x)$ point(s) de diffraction, de prédire la configuration d'équilibre en plan d'une plage abritée par un cap ou une structure artificielle. Dans le cas de la plage de San Lorenzo on note une bonne concordance de la ligne de côte à marée haute avec les prévisions du modèle théorique obtenues à partir de la direction du flux moyen d'énergie aux deux extrémités de la plage (voir figure 3).

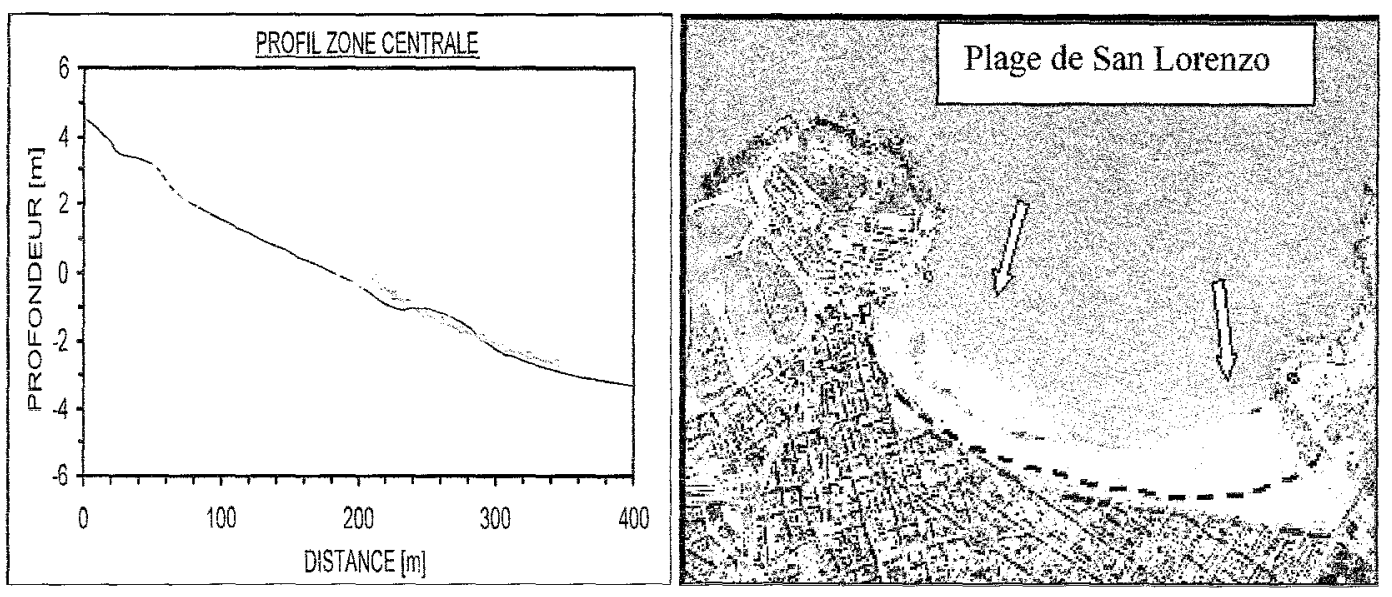

Figure 3. Configuration en profil et en plan de la plage de San Lorenzo

Figure 3. Cross section and plan view of San Lorenzo beach

\section{Influence de la digue Torres}

Il s'agit dans cette partie de déterminer dans quelle mesure la présence de la digue affecte la propagation de la houle, pour ensuite déterminer son impact sur l'équilibre de la plage.

\subsection{Impact sur la houle et le système de courants}

En procédant aux mêmes types de simulations que pour la situation actuelle, il apparait que la digue Torres affecte la propagation de la houle quelle que soit la direction d'incidence de celle-ci : elle provoque principalement une réduction de la hauteur de vague et une déviation des fronts d'onde par diffraction.

Sur la plage de San Lorenzo, l'effet de réduction de l'énergie est important, de même que l'effet de déviation des fronts qui affecte la direction du flux moyen d'énergie local. Par ailleurs, la présence de la nouvelle digue entraîne la disparition de 1'effet de concentration de la houle au niveau de la péninsule de Santa Catalina, disparition liée au bas-fond des Amosucas. Le système de courants, moins actif, est également modifié dans sa configuration : le flux de 
sortie, qui se trouve en situation actuelle au centre de la plage, se déplace vers l'est. Cette translation du flux de sortie n'apparait pas dans le cas des houles du N.-E, commence à se manifester dans le cas des houles du N. et du N./N.-E. et est importante dans le cas des houles du N.-O. Dans ce dernier cas, le système de courants n'est plus formé par deux boucles avec sortie au centre de la plage mais par un courant unique qui va de l'embouchure de la rivière Pilés à la péninsule de Santa Catalina.

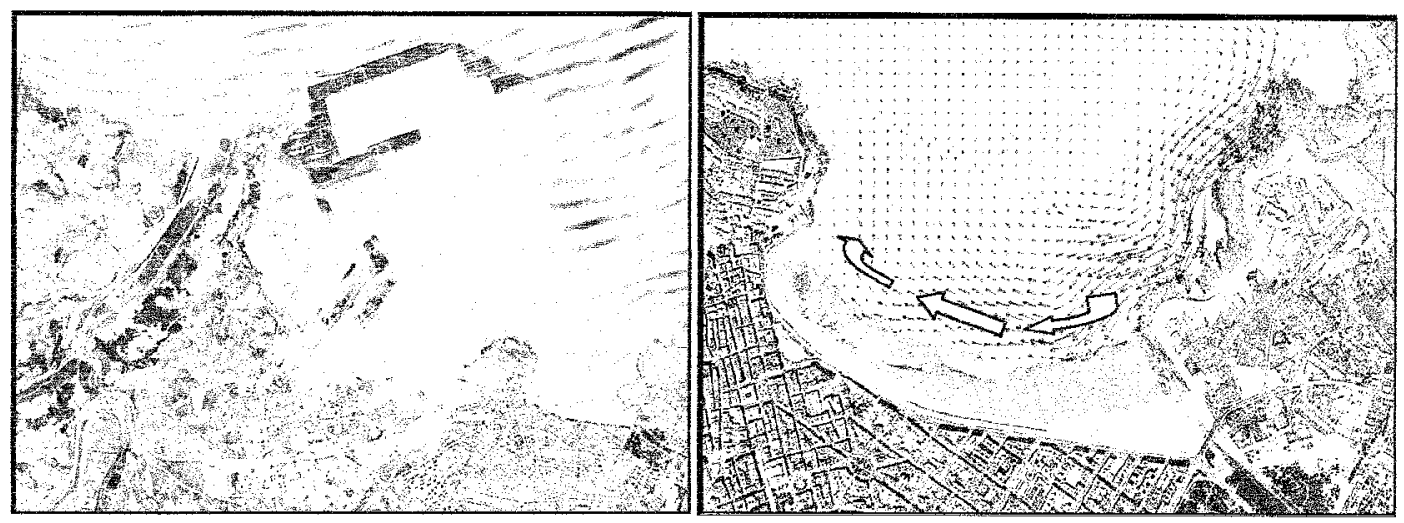

Figure 4. Exemple de propagation d'une houle du N.-O. et système de courants associé sur la plage de San Lorenzo (après la construction de la digue)

Figure 4. Example of $N W$ wave propagation and wave-induced currents on San Lorenzo beach (after the construction of the breakwater)

\subsection{Impact sur la dynamique littorale}

La modification des caractéristiques de la houle incidente due à la présence de la digue va entraîner des changements dans la configuration de la plage de San Lorenzo, aussi bien en profil qu'en plan. Ces changements consistent en une augmentation de la pente du profil de plage due à la diminution de la hauteur de vague et une rotation de la ligne de côte dans le sens horaire liée à la diffraction des fronts d'onde.

L'intensité de ces changements dépendant du volume de sédiments présent sur le site, deux situations peuvent être envisagées (Garcia, 2001).

Si on considère la simple redistribution du sable existant actuellement sur la plage, la rotation de la ligne de côte va se caractériser par un recul de $40 \mathrm{~m}$ dans la zone est et une avancée de $30 \mathrm{~m}$ dans la zone ouest, sans que soit modifiée la surface totale de plage sèche.

On peut d'autre part envisager d'apporter une quantité de sable telle que la rotation de la plage n'entraîne le recul d'aucun point de la ligne de côte, autrement dit envisager une avancée nette du front de plage par ajout de sable. Le volume nécessaire pour aboutir à cette configuration dépend de la prise en compte ou non de l'augmentation prévue de la pente du profil due à la réduction de l'énergie incidente. Si on ne prend pas en compte le changement de pente, le volume de sable à apporter serait de $150000 \mathrm{~m}^{3}$. Ce matériau devra avoir des caractéristiques similaires à celles du sable existant actuellement sur la partie de profil émergée $\left(\mathrm{D}_{50} \approx 0,35 \mathrm{~mm}\right)$. La rotation de la ligne de côte due à la présence de la nouvelle 
digue, couplée à cet apport de sable, permet d'obtenir une augmentation de la largeur de plage sèche de $30 \mathrm{~m}$ dans la zone ouest.

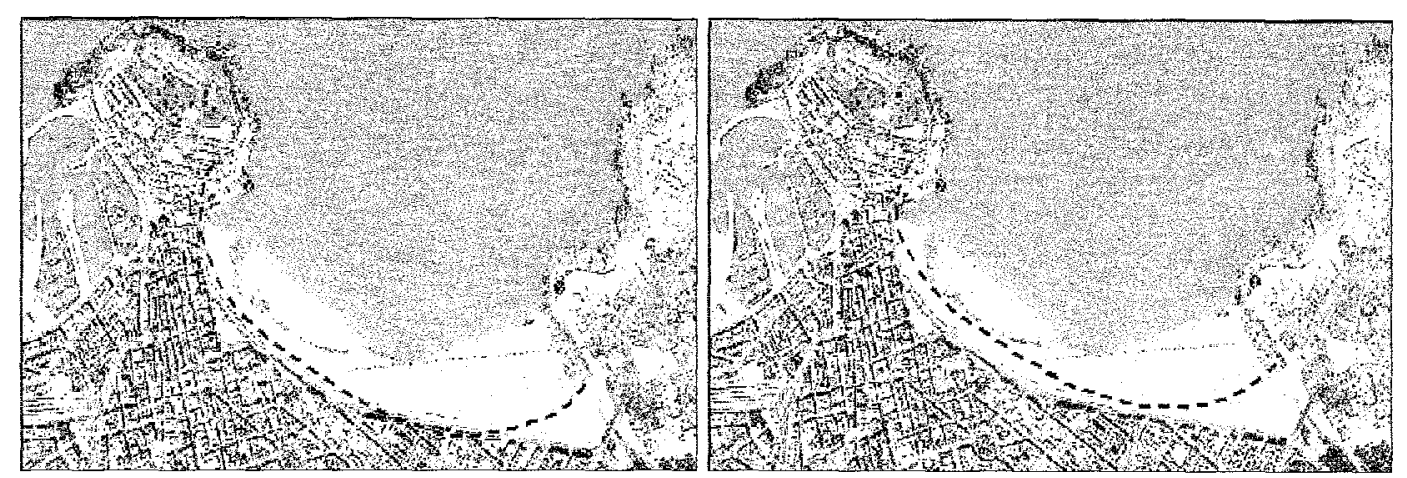

Figure 5. Configuration en plan de la plage de San Lorenzo après la construction de la digue, avec (gauche) et sans (droite) apport extérieur de matériel Figure 5. Plan view of San Lorenzo beach after the construction of the breakwater, without (left) and with (right) beach nourishment

\section{Conclusions}

L'analyse de la morphodynamique de la plage de San Lorenzo met en évidence que la plage, dans sa configuration actuelle, est en équilibre. Il existe cependant un déficit de plage sèche à marée haute, dans les parties centrale et occidentale de l'anse de San Lorenzo, préjudiciable à l'activité touristique. La construction de la digue d'agrandissement du port du Musel, en modifiant la propagation de la houle, va affecter cet équilibre. Les principales conséquences de la présence de l'ouvrage sur la dynamique de la zone sont une diminution de la hauteur de vague et une rotation des fronts d'onde par diffraction. Ces changements dans la propagation de la houle vont se manifester sur la plage de San Lorenzo par une augmentation de la pente du profil et une rotation de la ligne de côte dans le sens horaire. Ce changement de configuration, s'il est accompagné d'un apport extérieur de sable de $150000 \mathrm{~m}^{3}$, permet d'augmenter sensiblement la largeur de plage sèche et d'améliorer ainsi la fonctionnalité de la plage.

\section{Références}

R. MEDINA, A.M. BERNABEU, C. VIDAL, M. GONZALEZ (2000) Relationship between beach morphodynamics and equilibrium profiles - Proc. $27^{\text {th }}$ Int. Coastal Eng. Conference. A.S.C.E., Vol. 3, pp. 2589-2600.

M. GONZALEZ, R. MEDINA (2001) - On the application of static equilibrium bay formulations to natural and man-made beaches - Coastal Engineering, Ed. Elsevier, 43(2001), pp. 209-225.

N. GARCIA (2001) - Análisis del impacto en la dinámica litoral provocado por las obras de ampliación del Puerto del Musel, Gijón - Tesina de Master, Universidad de Cantabria. 